\title{
Sensitivity of the Overturning Circulation in the Southern Ocean to Decadal Changes in Wind Forcing
}

\author{
MiChAEL P. MEREDITH \\ British Antarctic Survey, Cambridge, United Kingdom \\ Alberto C. Naveira Garabato \\ National Oceanography Centre, Southampton, United Kingdom \\ ANDREw MCC. HogG \\ The Australian National University, Canberra, Australia \\ RICCARDO FARNETI \\ Earth System Physics Section, ICTP, Trieste, Italy
}

(Manuscript received 15 November 2010, in final form 15 June 2011)

\begin{abstract}
The sensitivity of the overturning circulation in the Southern Ocean to the recent decadal strengthening of the overlying winds is being discussed intensely, with some works attributing an inferred saturation of the Southern Ocean $\mathrm{CO}_{2}$ sink to an intensification of the overturning circulation, while others have argued that this circulation is insensitive to changes in winds. Fundamental to reconciling these diverse views is to understand properly the role of eddies in counteracting the directly wind-forced changes in overturning. Here, the authors use novel theoretical considerations and fine-resolution ocean models to develop a new scaling for the sensitivity of eddy-induced mixing to changes in winds, and they demonstrate that changes in Southern Ocean overturning in response to recent and future changes in wind stress forcing are likely to be substantial, even in the presence of a decadally varying eddy field. This result has significant implications for the ocean's role in the carbon cycle, and hence global climate.
\end{abstract}

\section{Introduction}

The Southern Ocean plays a disproportionately important role in determining global climate, in no small part because of its strong meridional overturning circulation and the associated fluxes and air-sea exchanges of heat, freshwater, and climatically important tracers such as $\mathrm{CO}_{2}$ (Le Quéré et al. 2007; Solomon et al. 2007). The strong westerly winds that overlie the Southern Ocean play a major role in driving this overturning circulation, and also the large horizontal transport of the Antarctic Circumpolar Current (ACC). These winds have

Corresponding author address: Michael P. Meredith, British Antarctic Survey, High Cross, Madingley Rd., Cambridge CB3 0ET, United Kingdom.

E-mail:mmm@bas.ac.uk strengthened in recent decades, due at least partly to anthropogenic processes (Thompson and Solomon 2002; Marshall 2003); however, observations and models indicate that the ACC transport is relatively insensitive to such changes (Meredith et al. 2004; Hallberg and Gnanadesikan 2006; Böning et al. 2008). The relative invariance of the ACC transport has been attributed to eddy saturation, whereby additional energy imparted from the winds is cascaded to the oceanic mesoscale instead of inducing prolonged accelerations of the horizontal mean flow (Hallberg and Gnanadesikan 2006; Meredith and Hogg 2006). Mesoscale eddies also exert important influences on the meridional overturning circulation of the Southern Ocean. Theories of this circulation (e.g., Marshall and Radko 2003; Olbers and Visbeck 2005) describe two contributions to the net overturning, with a directly wind-driven Eulerian mean cell 
that is counteracted partly by an opposing eddy-induced circulation (i.e., eddy compensation); the balance determines the residual overturning. The Eulerian mean overturning is concentrated in the near-surface layer and below the level of shallowest topography, while the eddy-induced component, assumed to be directed along isopycnals, will generate flow at both surface and intermediate levels.

A key question regarding the Southern Ocean is the extent to which the circumpolar and overturning circulations are linked. Or, equivalently, does an eddy-saturated circumpolar transport imply complete eddy compensation of the overturning? Zonally averaged theories (e.g., Marshall and Radko 2003) result in a closely coupled relationship between circumpolar and overturning transport, while Böning et al. (2008) assume the existence of such a relationship. Since circumpolar transport may be estimated (to first order) by isopycnal slope, while the overturning is more difficult to measure, such a constraint would help to evaluate changes in the overturning. In this paper, we investigate an ocean at (or close to) the eddy-saturated limit, and evaluate how the overturning circulation will behave at this limit.

The overall response of the overturning circulation in the Southern Ocean to changes in wind stress forcing will depend on the differing responses of the Eulerian mean and eddy-induced components (Fig. 1). The magnitude of the Eulerian mean overturning is reasonably expected to be linear with wind stress, particularly on short (intra annual) time scales (Ito et al. 2010), and is generally well represented in climate-scale ocean models (e.g., Fyfe and Saenko 2006; Sen Gupta and England 2006). The extent to which the eddy-induced overturning can compensate for changes in the Eulerian mean circulation on decadal time scales is much less clear, and advances using eddy-resolving models have long been hampered by computational constraints. Nonetheless, some progress has been made, for example Hallberg and Gnanadesikan (2006) conducted model experiments at a range of resolutions from coarse to eddy permitting, and showed that in the latter (and in contrast to the former) a significant fraction of the Ekman transport changes are compensated by eddyinduced transport drawing from lighter waters than does the mean overturning. More recently, Farneti et al. (2010) analyzed the Southern Ocean response to anomalous surface forcings in coarse and eddy-permitting versions of the National Oceanic and Atmospheric Administration (NOAA) Geophysical Fluid Dynamics Laboratory (GFDL) climate model, and found that in the eddypermitting integrations the eddies act as a buffer to atmospheric changes, with a reduced Southern Ocean response leading to weaker modifications in residual overturning circulation and ACC transport. Treguier et al.

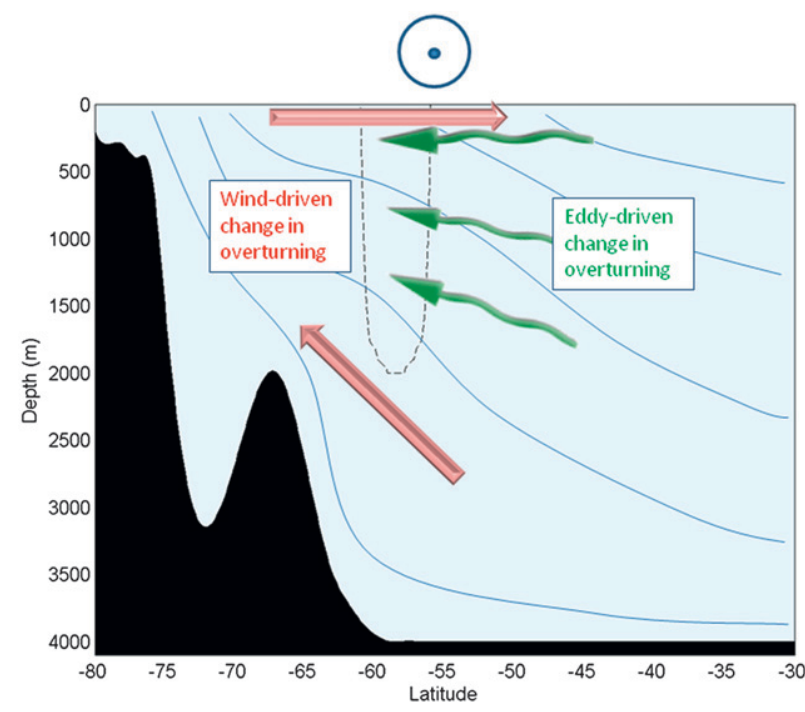

FIG. 1. Schematic of the response of the two components of the upper cell of the Southern Ocean overturning circulation to changing wind forcing (here shown for a positive increase in eastward wind stress). Directly wind-driven changes in overturning occur in the surface Ekman layer and below the layer of shallowest topography (the region of circumpolar flow unblocked by topography is bounded by the dashed line). An increase in the eddyinduced overturning circulation has the opposite sign, but with a different vertical structure, including a component in the region of flow unblocked by topography. Note that elements of the upper cell of the overturning circulation unrelated to changes in wind forcing are omitted for clarity.

(2010) used a global eddy-permitting model integrated over three decades, and highlighted that the zonal circulation and eddy kinetic energy in the Southern Ocean of their model were governed by different mechanisms on interannual versus decadal time scales, though they stressed that their model was not run to thermodynamic equilibrium and that this may be significant, with longer runs being necessary.

Focusing on the upper cell of the overturning circulation (where dense water is converted into lighter water), we here develop and test a new scaling for the sensitivity of eddy-induced mixing to changes in winds, and demonstrate that the observed invariance in the slopes of density surfaces implies that the overturning circulation in the Southern Ocean will vary as winds change, even in the presence of a decadally varying eddy field. Our results show that eddy saturation and eddy compensation are dynamically distinct phenomena; thus, occurrence of one does not necessarily imply the other. We demonstrate that the Southern Ocean overturning is very likely to increase in response to recent and future climate change, with significant implications for the ocean's role in the carbon cycle, and hence global climate. 


\section{The models and experiments}

To investigate the response of eddy activity to changes in winds, we conduct simulations using a quasigeostrophic coupled ocean-atmosphere model with a three-layer ocean (Q-GCM; Hogg et al. 2003), here run in uncoupled mode. Wind stress is zonal and constant, with a simple maximum in the center of the domain (a zonally reentrant channel, $23040 \times 2880 \mathrm{~km}$, with walls on the meridional boundaries). The model uses topography from Smith and Sandwell (1997), truncated at $\pm 900 \mathrm{~m}$ for consistency with the quasigeostrophic assumptions. Numerical parameters are such that the horizontal resolution $(10 \mathrm{~km})$ is significantly smaller than the first Rossby radius $(43 \mathrm{~km}$ ) to ensure resolution of baroclinic eddies. A vigorous eddy field is ensured by using low viscosity (biharmonic with a coefficient of $3 \times 10^{10} \mathrm{~m}^{4} \mathrm{~s}^{-1}$ ). Under such conditions, this model has been shown to generate an eddy-saturated state (Meredith and Hogg 2006).

Quasigeostrophic models are unable to generate diapycnal flow and so are not suitable for direct investigations of Southern Ocean overturning; instead, this is done here using the GFDL Climate Model, version 2.4 (CM2.4), of which the ocean component is the Modular Ocean Model, version 4 (MOM4; Griffies et al. 2005). $\mathrm{CM} 2.4$ uses a square isotropic grid with oceanic resolution of $1 / 4^{\circ}$ (equivalent to $13.8 \mathrm{~km}$ at $60^{\circ} \mathrm{S}$ and $9 \mathrm{~km}$ at $\left.70^{\circ} \mathrm{S}\right)$. Furthermore, the ocean model does not use a parameterization of mesoscale eddy mixing, allowing the resolved flow to operate in the absence of parameterized eddies. Following a control simulation, two perturbation simulations were performed (Farneti et al. 2010; Farneti and Delworth 2010). In the first, $\mathrm{CO}_{2}$ concentrations were increased by $1 \% \mathrm{yr}^{-1}$ until doubled, and then held fixed (experiment CC); this featured time-varying mechanical and buoyancy forcings. In the second, a temporally invariant anomalous wind stress pattern was added to the wind stress felt by the ocean between $20^{\circ}$ and $75^{\circ} \mathrm{S}$, resulting in a doubling of wind stress felt by the ocean (experiment WIND). Similar experiments are conducted with the coarse-resolution version of the GFDL Coupled Model, version 2.1 (CM2.1), which features an oceanic resolution of $1^{\circ}$ and employs a parameterization for eddy-induced advection and along-isopycnal diffusion of tracers (Gent and McWilliams 1990, hereafter GM).

The eddy-permitting GFDL CM2.4 model is in remarkably good agreement with observations in terms of eddy kinetic energy (EKE) and its ACC transport is around $190 \mathrm{~Sv}\left(1 \mathrm{~Sv} \equiv 10^{6} \mathrm{~m}^{3} \mathrm{~s}^{-1}\right)$, which is somewhat higher than observational estimates (Farneti et al. 2010). Since we focus here on the decadal time scale, results are computed as time means for years $36-40$ of the
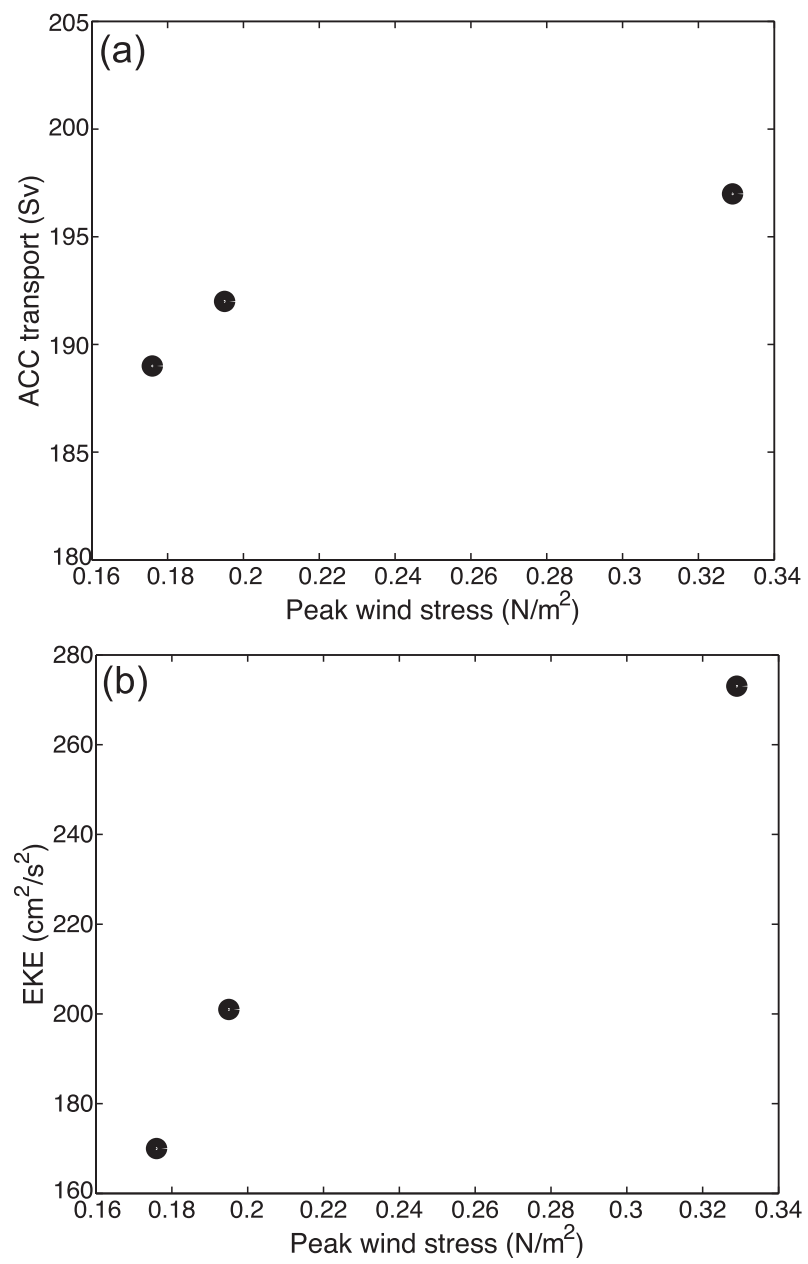

FIG. 2. (a) ACC transport (Sv) and (b) EKE $\left(\mathrm{cm}^{2} \mathrm{~s}^{-2}\right)$ as a function of peak wind stress for the GFDL CM2.4 model in the perturbation simulations. The EKE has been averaged in the $60^{\circ}-$ $45^{\circ} \mathrm{S}$ latitude band and values are computed as time means for years 36-40 after the perturbation runs are started. In this model, the ACC transport is nearly independent of wind stress forcing (less than a 10-Sv increase for a near doubling of wind stress) and EKE shows a quasi-linear dependency on wind stress.

perturbation runs. Eddy-induced overturning is calculated as the maximum value at the location of the maximum change in Eulerian mean circulation (around $50^{\circ} \mathrm{S}$ ), which corresponds to the maxima below the mixed layer. In the CM2.4 model, ACC transport is found to be nearly independent of wind stress forcing, with a change in transport of $<10 \mathrm{~Sv}$ for a near-doubling of peak wind stress (Fig. 2a). EKE has a much stronger sensitivity, with a quasi-linear dependency on wind stress (Fig. 2b). This model thus reproduces a Southern Ocean that is near the eddy-saturation limit.

The eddy-permitting resolution of CM2.4 is certainly not adequate to resolve all eddy baroclinic modes, and refining the horizontal resolution might modify the eddy 
(a)

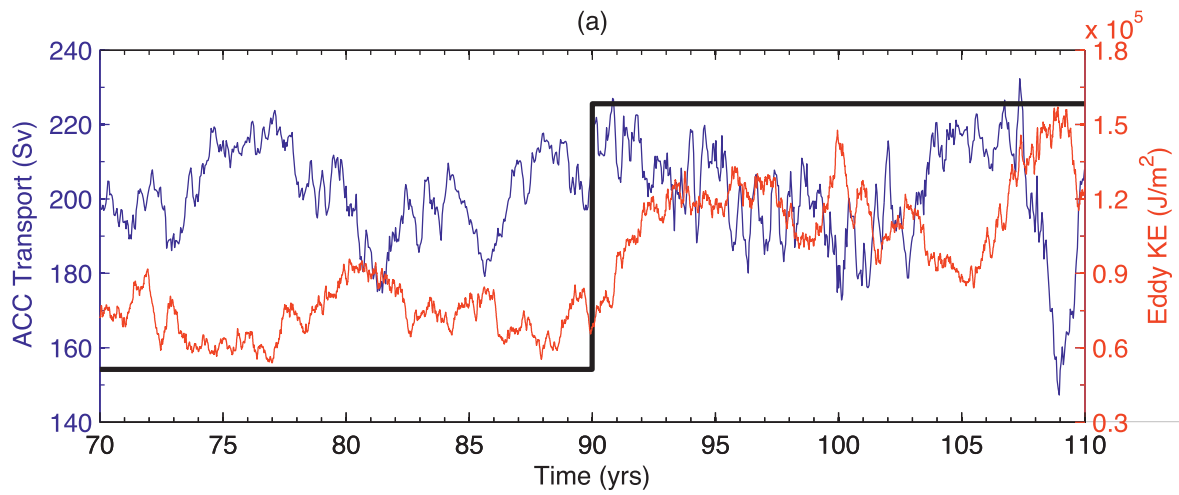

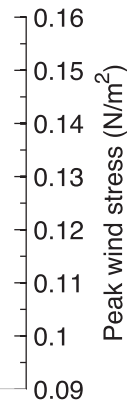

(c)
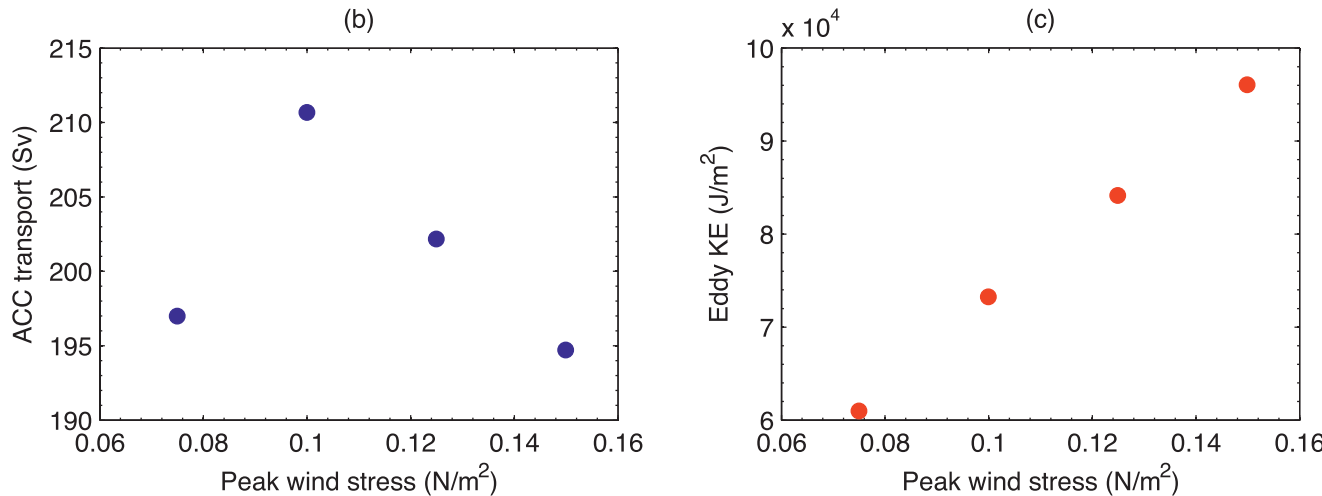

FIG. 3. Results from Q-GCM demonstrating the linear dependence of EKE upon wind stress in the eddy-saturated limit. (a) Time series of ACC transport (blue) and EKE (red) over $40 \mathrm{yr}$ of a simulation. The initial value of peak wind stress is $0.1 \mathrm{~N} \mathrm{~m}^{-2}$; this is increased to $0.15 \mathrm{~N} \mathrm{~m}^{-2}$ at model year 90 as shown by the black line. EKE increases with a short lag, while transport changes are indistinguishable from existing variability. (b) ACC transport and (c) $\mathrm{EKE}$ as a function of peak wind stress. Averages over 60 model years of an equilibrium state are shown.

response to surface forcings. Accordingly, and to support the adequacy of the CM2.4 results, we also make use of the eddy-resolving solutions of Viebahn and Eden (2010), who used an idealized ACC configuration of the CPFLAME model (one of the Family of Linked Atlantic Model Experiments) with a zonally reentrant channel connected to an idealized Atlantic basin. This model was run with 5-km horizontal resolution, and wind perturbation experiments were conducted from which the eddy diffusivity $\kappa$ was computed directly.

\section{A scaling estimate of the Southern Ocean overturning}

Theory and models (Straub 1993; Hallberg and Gnanadesikan 2006; Meredith and Hogg 2006) have indicated that at the eddy-saturation limit, EKE increases with changes in wind stress; this prediction is consistent with observations on the circumpolar (Meredith and Hogg 2006) and regional scales (Morrow et al. 2010), albeit on interannual rather than decadal time scales. Figure 3 a shows a multidecadal simulation using Q-GCM, in which wind stress is increased by $50 \%$ at model year 90; the resulting increase in EKE is unequivocal, but no change above internal variability is observable for the ACC transport. The EKE response was determined for a wide range of wind stress changes, revealing an approximately linear relationship (Fig. 3c), with no trend in ACC transport (Fig. 3b). The Q-GCM results are consistent with the behavior shown by the GFDL CM2.4 coupled model (Fig. 2).

An understanding of the implications of such a linear response of EKE for the overturning circulation requires knowledge of the dependence of the eddy-induced overturning on the eddy field. A complete theory for this does not exist, but progress is made here by developing some novel and relatively simple theoretical scaling arguments. First we consider the relationship between the eddy-induced overturning streamfunction $\Psi^{*}$ and the isopycnal eddy diffusivity $\kappa$, which quantifies the rate at which mesoscale eddies homogenize potential vorticity $(q)$ along isopycnal surfaces (strictly isentropic surfaces, but taken here to be equivalent):

$$
\Psi_{z}^{*}=-\frac{\kappa q_{y}}{f},
$$


(a) GFDL/CM2.4 CTL run (PV)

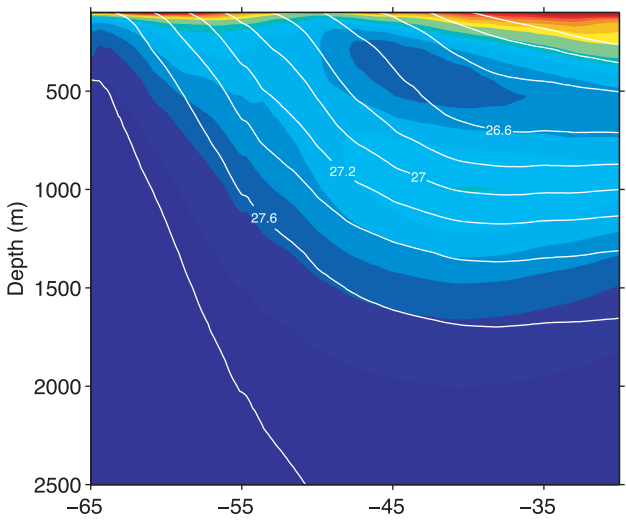

(c) GFDL/CM2.4 CTL run (PVG)

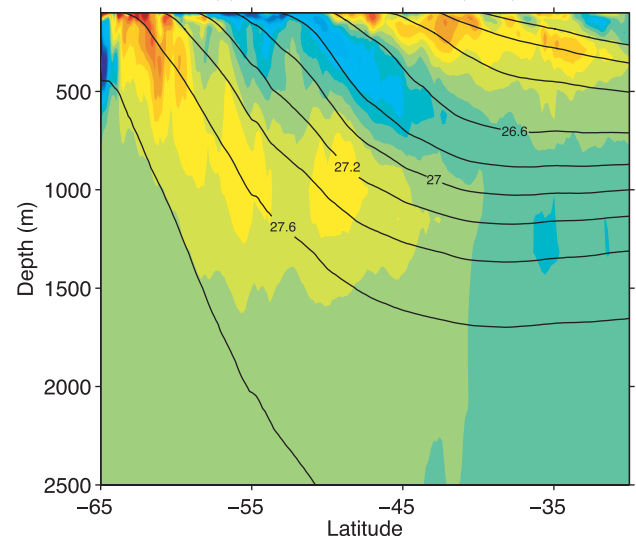

(b) GFDL/CM2.4 WIND run (PV)

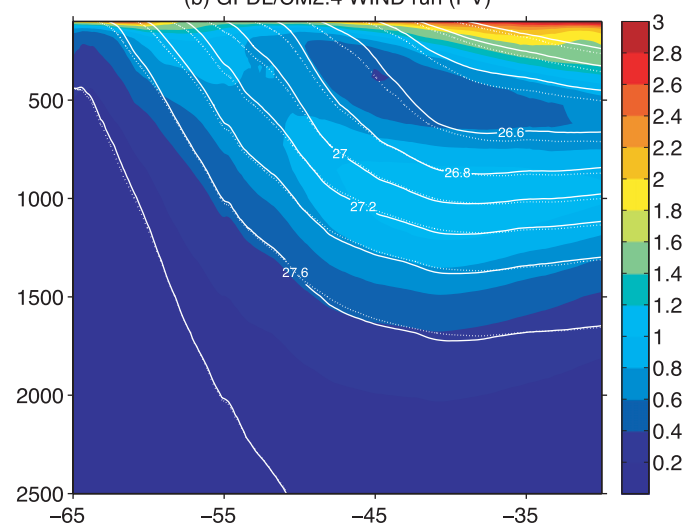

(d) GFDL/CM2.4 WIND run (PVG)

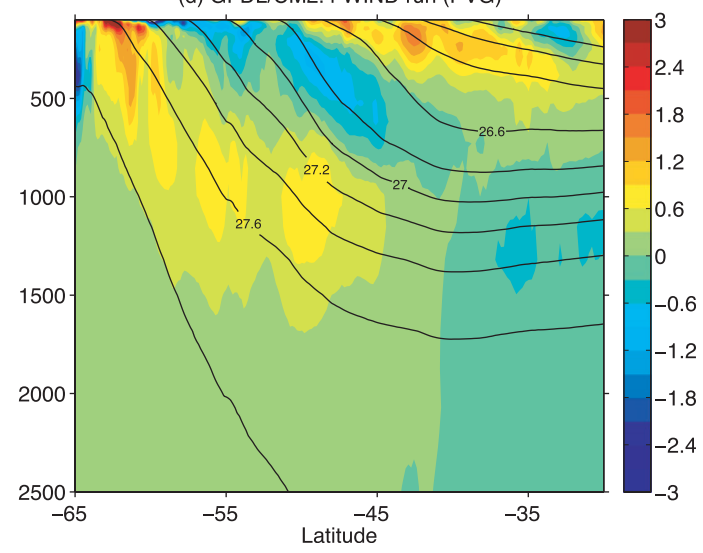

FIG. 4. Zonal-mean potential vorticity $\left[(\mathrm{m} \mathrm{s})^{-1} \times 10^{-10}\right.$ and the sign is reversed for clarity $]$ and its meridional gradient $\left(\mathrm{m}^{-2} \mathrm{~s}^{-1} \times 10^{-16}\right)$ in the GFDL CM2.4 model. The potential vorticity is defined here as $-(f / \rho) \partial_{z} \sigma_{0}$, where $f$ is the Coriolis parameter, $\rho$ is in situ density, $\sigma_{0}$ is the potential density referenced to the surface, and we have neglected relative vorticity. (a) Time-mean potential vorticity (PV) in the control run (CTL); also shown are the mean isopycnals (white contours, interval $0.2 \mathrm{~kg} \mathrm{~m}^{-3}$ ). (b) Potential vorticity in the wind perturbation experiment (WIND), averaged for the 36-40-yr period after the beginning of the perturbation; also shown are the mean isopycnals for the same period (solid white lines), together with the CTL isopycnals (dotted white lines). (c),(d) As in (a),(b), but for the meridional gradient of potential vorticity (PVG). The upper $100 \mathrm{~m}$ have been excluded in all plots.

where $f$ is the Coriolis parameter, $q$ is the Ertel potential vorticity, subscripts are used to represent partial derivatives, and we have assumed that thickness fluxes dominate potential vorticity fluxes and an $f$ plane (Vallis 2006).

This relationship applies in the largely adiabatic ocean interior, and describes the proportionality of the eddyinduced meridional velocity (to which the upwelling rate is related through continuity) to the eddy flux of potential vorticity along isopycnals. Potential vorticity depends primarily upon stratification, which has been observed (albeit by relatively sparse measurements) to be insensitive to wind forcing (Böning et al. 2008); consequently, the potential vorticity distribution of the Southern Ocean interior can be assumed to be largely insensitive to decadal-scale changes in wind forcing. This is demonstrated further here using the GFDL CM2.4 model by computing the potential vorticity and its meridional gradient for the Southern Ocean under the different wind forcing scenarios. We define here potential vorticity as $-(f / \rho) \partial_{z} \sigma_{0}$, where $f$ is the Coriolis parameter, $\rho$ is in situ density, $\sigma_{0}$ is potential density referenced to the surface, and we have neglected relative vorticity. In Fig. 4 we show the zonal-mean potential vorticity and its meridional gradient in the GFDL CM2.4 model for both the control (CTL) and wind-perturbation (WIND) experiments, averaged for the $36-40-\mathrm{yr}$ period after the beginning of the perturbation. For roughly a doubling of wind stress over the Southern Ocean region, the eddypermitting model CM2.4 shows little change in isopycnal slope (white contours in Figs. 4a,b), potential vorticity structure, and overall meridional gradient in potential vorticity (Figs. 4c,d). Hence, in this model, the Southern 
(a) GFDL/CM2.1 CTL run (PV)

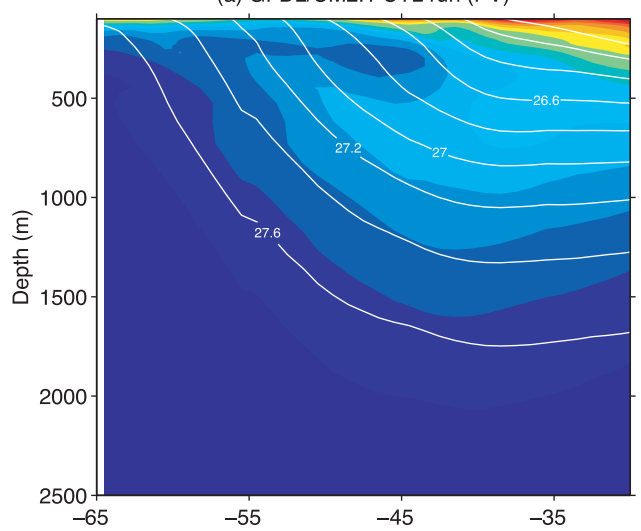

(c) GFDL/CM2.1 CTL run (PVG)

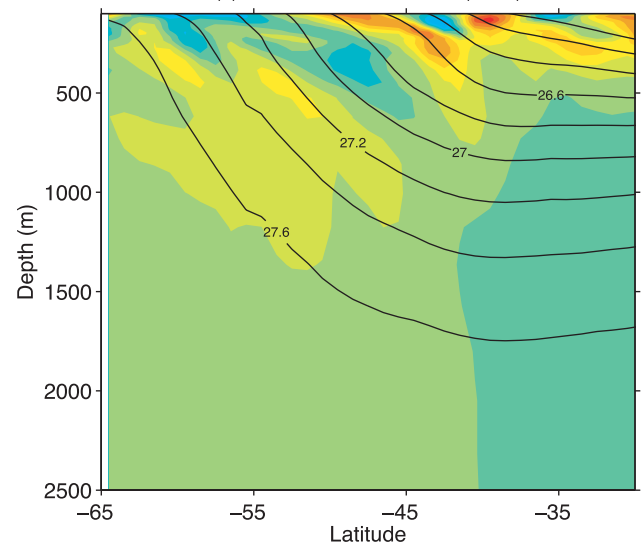

(b) GFDL/CM2.1 WIND run (PV)

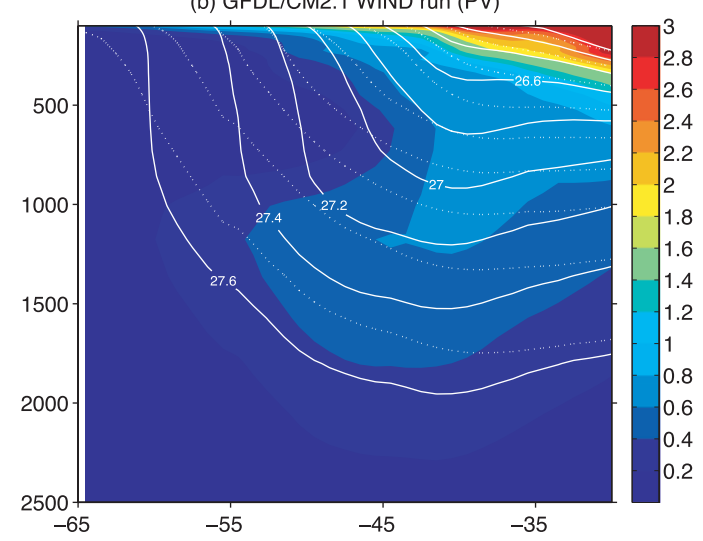

(d) GFDL/CM2.1 WIND run (PVG)

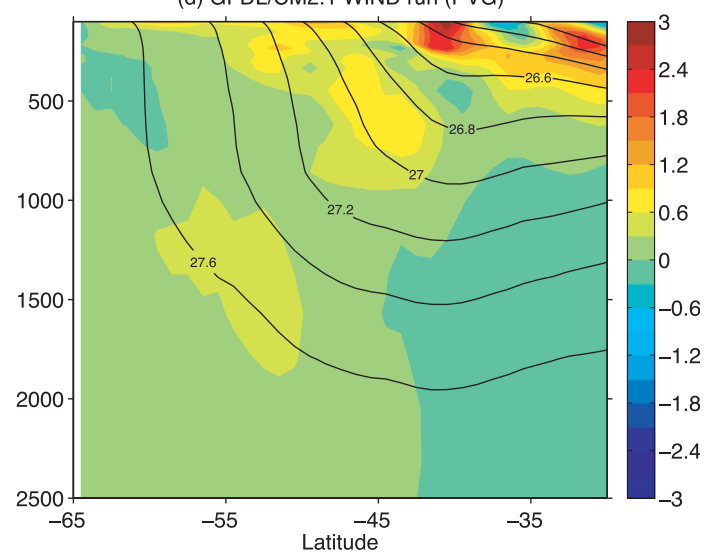

FIG. 5. As in Fig. 4, but for the GFDL CM2.1 model. Note the large sensitivity of the coarse-resolution model to changing winds evidenced by the (b) steepening of isopycnals and (c),(d) large modifications of meridional gradients of PV.

Ocean gradient of potential vorticity is approximately constant, and independent of forcing strength. This is not true for coarse-resolution coupled models where mesoscale eddies have to be parameterized. Results from GFDL CM2.1 show large changes in isopycnal slope and potential vorticity gradients (Fig. 5). As discussed in Farneti and Gent (2011), closures for the eddy-induced advection coefficient that are presently used in coarseresolution climate models are inadequate to accurately represent the eddy sensitivity to wind stress changes, and their results are therefore compromised for a significant increase in the zonal winds over the ACC.

If the potential vorticity distribution of the Southern Ocean interior is independent of decadal changes in wind forcing, the problem reduces to assessing how $\kappa$ responds to changes in wind forcing. Recent investigations of eddy-induced mixing across a broad, parallel oceanic jet (Ferrari and Nikurashin 2010; Naveira Garabato et al. 2011.) have shown that the evolution of a passive tracer advected by the jet and a nonlinear eddy field may be characterized by

$$
\kappa=\frac{\kappa_{\text {Taylor }}}{\left[1+\gamma^{-2} k^{2}\left(U_{m}-c\right)^{2}\right]},
$$

where

$$
\kappa_{\text {Taylor }}=\frac{k^{2}}{\left(k^{2}+l^{2}\right)} \frac{\text { EKE }}{\gamma}
$$

is Taylor's definition of the eddy diffusivity in the absence of a mean flow (Taylor 1921), $\gamma$ is a linear eddy damping rate mimicking the nonlinear damping of each eddy through interaction with other eddies in the ocean, $(k, l)$ is the horizontal wave vector of the monochromatic eddy field, $U_{m}$ is the mean flow speed, and c is the eddy phase speed. Assuming that $l<k$ and that $U_{m}>c$, as 
commonly observed in the Southern Ocean, (2) may be approximated as

$$
\kappa=\frac{\mathrm{EKE}}{\gamma\left(1+\gamma^{-2} k^{2} U_{m}^{2}\right)} .
$$

Following the tenet that the decorrelation time scale of eddies of length scale $k^{-1}$ scales with the eddy strain rate in two-dimensional turbulent flows (Salmon 1998), it has been shown that taking $\gamma^{-2} k^{2} \approx 4 /$ EKE provides a good fit between (3) and altimetry-based "effective diffusivity" calculations in the surface Southern Ocean (Ferrari and Nikurashin 2010). Accordingly, (3) reduces to

$$
\kappa \propto \frac{\mathrm{EKE}^{1 / 2}}{k\left(1+4 U_{m}^{2} / \mathrm{EKE}\right)} .
$$

For large $U_{m}$ or small EKE, (4) approximates to

$$
\kappa \propto \frac{\mathrm{EKE}^{3 / 2}}{4 k U_{m}^{2}} .
$$

For small $U_{m}$ or large EKE, (4) instead approximates to

$$
\kappa \propto \frac{\mathrm{EKE}^{1 / 2}}{k} .
$$

It follows that, in the absence of significant temporal changes in $U_{m}$ (as suggested by the observed statistically stationary ACC transport), the dependence of $\kappa$ on EKE ranges from a power of $1 / 2-3 / 2$, encompassing the linear regime. Given the linear relationship between winds and EKE at the eddy-saturation limit (Figs. 2 and 3), $\kappa$ can be expected to have approximately the same dependence on wind stress. Thus, it is possible to map out a region of predicted change in $\kappa$ for a given change in winds; this is shaded in Fig. 6.

Following (1) and (4), and recognizing the general insensitivity of the potential vorticity structure of the Southern Ocean interior to decadal changes in forcing (Böning et al. 2008; see also Fig. 4), we obtain the result that changes in the rate of eddy-induced upwelling scale with the wind stress with a power of $1 / 2-3 / 2$, again encompassing the linear regime. Under these circumstances, the shaded area in Fig. 6 translates directly to the predicted change in eddy-induced upwelling for a given change in wind stress.

Our predicted change in $\kappa$ with wind stress can be considered relative to two limiting cases. The first is zero compensation (solid black line in Fig. 6), in which eddyinduced overturning is invariant with respect to winds.

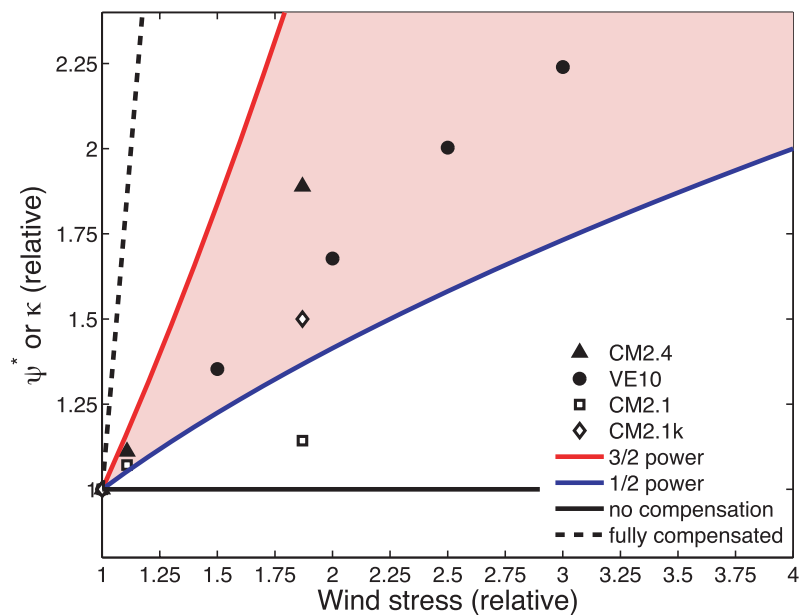

FIG. 6 . The area bounded by powers of $1 / 2$ and $3 / 2$ (blue and red lines) covers the region of predicted change in $\kappa$ for a given change in winds. Recognizing the general insensitivity of the potential vorticity structure of the Southern Ocean interior to decadal changes in forcing (Böning et al. 2008), the displayed change in $\kappa$ is equivalent to the resultant change in eddy-induced overturning $\left(\Psi^{*}\right)$. The black lines show the limiting cases of no eddy compensation (solid) and complete eddy compensation (dashed). Results of eddy-permitting (CM2.4, perturbation experiments $\mathrm{CC}$ and WIND; triangles) and eddy-resolving [Viebahn and Eden 2010 (VE10); circles] primitive equation models are consistent with the predicted sensitivity of eddy-induced overturning to winds derived here. As a result of poor representation of eddy-induced transports, our coarse-resolution model lies outside the region of predicted change (CM2.1; squares). A CM2.1 integration with modifications to the GM parameters (CM2.1k; diamond) still has a weaker response than CM2.4, but now lies within the theoretical bounds; however, this configuration introduces worsened climatological biases and other problems.

The second is where complete compensation occurs; that is, $\kappa$ increases sufficiently to exactly counter the increase in the Eulerian mean component of the overturning. To estimate the sensitivity of $\kappa$ to changes in wind stress at this limit, we consider the zonal momentum equation for an outcropping isopycnal layer (see the appendix for a full derivation):

$$
-\frac{\langle f v h\rangle}{\langle f\rangle\langle h\rangle}=\frac{\langle h\rangle\left\langle v^{\prime} q^{\prime}\right\rangle}{\langle f\rangle}-\frac{\langle\tau\rangle}{\left(\langle f\rangle \rho_{0}\langle h\rangle\right)}
$$

where $h$ is layer thickness, $\rho_{0}$ is a reference density, angled brackets indicate a quasi-zonal (streamwise) and temporal average, and primes denote deviations from that average. The eddy potential vorticity flux can then be parameterized as

$$
\left\langle v^{\prime} q^{\prime}\right\rangle \propto \kappa\left(-\frac{\beta}{\langle h\rangle}+\frac{f\langle h\rangle_{y}}{\langle h\rangle^{2}}\right),
$$


where $\beta$ is the meridional derivative of $f$ and an equivalence between the eddy diffusivities for passive and active (such as $q$ ) tracers has been assumed. Substituting (8) into (7), multiplying by $\langle h\rangle$ and taking small variations, we obtain

$$
\begin{aligned}
-\delta\left(\frac{\langle f v h\rangle}{\langle f\rangle}\right)= & \delta \kappa\left(-\frac{\beta\langle h\rangle}{f}+\langle h\rangle_{y}\right) \\
& +\kappa \delta\left(-\frac{\beta\langle h\rangle}{f}+\langle h\rangle_{y}\right)-\frac{\delta(\langle\tau\rangle)}{\langle f\rangle \rho_{0}}
\end{aligned}
$$

If the residual overturning is invariable, $\delta(\langle f v h\rangle /\langle f\rangle) \approx 0$, and if stratification remains constant (as suggested by observations), $\delta\left(-\beta\langle h\rangle / f+\langle h\rangle_{y}\right) \approx 0$. Reorganizing and dividing by $\kappa$ then gives

$$
\frac{\delta \kappa}{\kappa} \approx \frac{\langle\tau\rangle}{\kappa \rho_{0}\left(-\beta\langle h\rangle+f\langle h\rangle_{y}\right)} \frac{\delta(\langle\tau\rangle)}{\langle\tau\rangle} .
$$

Using characteristic values of $\beta=10^{-11} \mathrm{~m}^{-1} \mathrm{~s}^{-1}, \rho_{0}=$ $1000 \mathrm{~kg} \mathrm{~m}^{-3}, f=10^{-4} \mathrm{~s}^{-1}, \kappa=1000 \mathrm{~m}^{2} \mathrm{~s}^{-1},\langle\tau\rangle=$ $0.12 \mathrm{~N} \mathrm{~m}^{-2}$, and estimates of $\langle h\rangle$ and $\langle h\rangle_{y}$ appropriate to the Upper Circumpolar Deep Water layer in the Southern Ocean $\left(\langle h\rangle \sim 1000 \mathrm{~m},\langle h\rangle_{y} \sim 500 \mathrm{~m} / 2000 \mathrm{~km}\right)$, we obtain

$$
\frac{\langle\tau\rangle}{\kappa \rho_{0}\left(-\beta\langle h\rangle+f\langle h\rangle_{y}\right)} \sim 8 .
$$

Accordingly, we estimate that complete compensation would be achieved if

$$
\frac{\delta \kappa}{\kappa} \sim 8 \frac{\delta \tau}{\tau}
$$

giving the dashed black line in Fig. 6. Our shaded area lies within these lines, hence the scaling arguments presented here predict partial eddy compensation of the Southern Ocean overturning on decadal time scales of stratification invariance, and constrain the level of eddy compensation that is likely to occur.

To quantitatively test the applicability of the scaling, we use the GFDL CM2.4 model, which features a full primitive equation ocean model run at eddy-permitting resolution (Griffies et al. 2005; Farneti et al. 2010). In Fig. 6, the black triangles depict the sensitivity of the eddy-induced overturning to changes in wind stress in this model (perturbation experiments CC and WIND), and are seen to lie close to the center of the shaded area predicted by our scaling analysis. Moreover, the direct estimates of $\kappa$ from the eddy-resolving simulations of Viebahn and Eden (2010) also fall in between the two power laws (black circles in Fig. 6), adding more confidence to the numerical solutions and resolution dependence of our simulations. While such results may depend to some extent on the details of the models, they lend significant support to the applicability of the theoretical scaling.

Significantly, the coarse-resolution model CM2.1 does not reproduce the sensitivity of the eddy-induced overturning that is obtained when eddies are permitted or resolved: for significant changes in wind stress forcing, the GFDL CM2.1 model (open squares in Fig. 6) lies well outside the bounds of the predicted area. When the upper limit to the effect of parameterized eddies is changed to be an order of magnitude higher than in the original CM2.1 (Farneti and Gent 2011), the coarseresolution model has a stronger sensitivity to the changing winds and now falls within the region of predicted change (CM2.1k, open diamond in Fig. 6). However, as discussed in Farneti and Gent (2011), many fundamental problems persist in the $\mathrm{CM} 2.1 \mathrm{k}$ configuration as climatological biases are strengthened and the ACC sensitivity remains very high (i.e., no eddy saturation is achieved). Farneti and Gent (2011) concluded that numerical approaches for the parameterization of eddyinduced transports that are presently used in coarseresolution coupled models prevent them from faithfully representing the eddy response to significant changes in the forcing fields, and new and improved closures for $\kappa$ are needed and should be tested in coupled climate models. A depth-dependent formulation for $\kappa$ is the first step forward, and examples of efforts in this directions are given in Danabasoglu and Marshall (2007), Eden and Greatbatch (2008), and more recently in Hofmann and Morales Maqueda (2011) with promising results.

\section{Discussion}

It was argued previously that the observed decadal invariance of isopycnal slopes in the Southern Ocean is evidence of insensitivity of the overturning circulation to changes in winds (Böning et al. 2008), and it is true that energy and momentum budgets indicate that a directly wind-driven change in overturning must be compensated to a significant extent by an increase in the eddy-induced circulation (Olbers and Ivchenko 2001; Hallberg and Gnanadesikan 2006). However, for complete eddy compensation of the overturning circulation, changes in the magnitude of its two contributions would need similar vertical structures. This conflicts with our understanding of their dynamics, whereby the Eulerian mean overturning is intensified in the surface layer and below the depth of the major topography, while changes in the eddy-induced overturning will occur in any layers that have significant potential vorticity gradients. The most prominent among such layers are at intermediate depths 
(a few hundred to a thousand meters or more) that are largely unaffected by the Eulerian mean circulation (Speer et al. 2000; Marshall and Radko 2003), and a net change in the mass flux along these layers is expected if the potential vorticity distribution is invariant in time. Such invariance is implied by the observed persistence of isopycnal slopes in the Southern Ocean; hence, this observation is actually evidence of the sensitivity of overturning circulation to winds on decadal time scales. Support for this assertion derives from the results of eddypermitting ocean models (Hallberg and Gnanadesikan 2006; Farneti et al. 2010; Viebahn and Eden 2010), which show overturning circulations that are sensitive to changes in wind forcing at levels consistent with the ideas expressed here, including the above scaling analysis (Fig. 6). Of particular note is the finding that an approximate $20 \%$ increase in wind stress over the Southern Ocean in these models led to an increase of a similar magnitude in the overturning, with upwelling being focused in the intermediate layers where significant potential vorticity gradients exist (Hallberg and Gnanadesikan 2006; Farneti et al. 2010). We emphasize that the preceding discussion hinges on the assumption of a largely invariant potential vorticity field across the Southern Ocean. That this assumption appears to hold on time scales of decades likely relates to the much longer adjustment time of the ACC and global pycnocline, which has been suggested to be on the order of several centuries owing to the large volume of the tropical ocean (see discussion in Allison et al. 2011). We suggest that, on those centennial time scales, changes in the rate of potential vorticity homogenization by eddies associated with variations in EKE and wind stress in the Southern Ocean will significantly alter the region's potential vorticity distribution, and thereby lead to a different degree of compensation compared with that occurring on decadal time scales.

The sensitivity of the overturning in the Southern Ocean to changes in wind forcing is critical to recent and future climate change, not least because it exerts an important control on the rate at which $\mathrm{CO}_{2}$ enters or exits the ocean interior. On this matter, it was postulated recently that the Southern Ocean carbon sink has become saturated in recent decades (Le Quéré et al. 2007), and a debate ensued that focused on the size of the data network from which the saturation was inferred, and a possible future reversal of the saturation (Law et al. 2008; Zickfeld et al. 2008; Le Quéré et al. 2008). The postulated saturation of the Southern Ocean carbon sink was attributed by Le Quéré et al. (2007) to a windforced increase in net overturning of order a few Sverdrups. The possibility of a change of this magnitude was challenged by Böning et al. (2008) on the basis of an observed invariance of isopycnal slopes on decadal time scales; however, we have demonstrated here that this observed invariance and contemporary understanding of the dynamics of the ACC system are, in fact, consistent with a net increase in overturning having occurred, albeit at a slower rate than predicted by coarseresolution models.

In terms of the impact on the Southern Ocean carbon sink, it is important to note that the intermediate layers in which the eddy-induced overturning is likely to have increased most strongly are those where the concentration of dissolved inorganic carbon is largest (Key et al. 2004; Le Quéré et al. 2007). Accordingly, the role of this component of the overturning circulation is likely to be disproportionately important in controlling vertical carbon fluxes and the magnitude of the carbon sink. There are other significant implications relating to the response of the Southern Ocean to changing wind forcing as elucidated here. For example, the deep waters of the ACC are rich in macronutrients, and their vertical supply to the euphotic zone is a key factor in determining primary production in the Southern Ocean (e.g., Pollard et al. 2002), with consequences also for the other trophic levels of the ecosystem.

Our analysis has highlighted once more the need for new and improved closures for the isopycnal eddy diffusivity $\kappa$ in coarse-resolution climate models (see also the discussion in Farneti and Gent 2011). The proposed scaling has the potential to help and guide new parameterizations that, for example, assume $\kappa$ to be proportional to EKE and could thus produce a more dynamic and energetically consistent response to wind stress changes, as recently proposed by Eden and Greatbatch (2008) and Marshall and Adcroft (2010). Also, a new depth-dependent formulation for $\kappa$ (Danabasoglu and Marshall 2007) has been shown to respond much more readily to changes in wind forcing (Gent and Danabasoglu 2011), with partial eddy compensation of the overturning circulation that seems consistent with our scaling.

We note that we only considered here the effect of strengthening winds on the Southern Ocean dynamics. Of course, because of climate change, buoyancy fluxes also change significantly. It was noted in Farneti et al. (2010) but studied further in Hogg (2010), Treguier et al. (2010), and Morrison et al. (2011) that changes in buoyancy forcing do imply some thermodynamic adjustment that can offset the wind contribution to both circumpolar and overturning circulation. Hence, we suggest that inclusion of buoyancy effects in our theoretical and numerical results would further modify the eddy-induced response. 


\section{Conclusions}

In this paper, we have specifically considered the effect of strengthening winds on the Southern Ocean dynamics, given the observed changes in such winds and the intense speculation surrounding their consequences (Böning et al. 2008). We have placed theoretical constraints on the likely sensitivity of Southern Ocean overturning circulation to decadal-scale climatic changes in wind forcing, incorporating the role of eddies in directly counteracting wind-induced changes. Our scaling results predict that an eddy-saturated ocean will result in partial (but not full) eddy compensation, and models close to the eddy-saturated limit show sensitivity consistent with our theory. Further predictive skill is needed to narrow further the range of possible future overturning circulations: for now, the evolution of the Southern Ocean overturning and its impact on the carbon cycle remain critical research priorities.

Acknowledgments. The authors thank Steve Rintoul, Kevin Speer, Harry Bryden, Corinne Le Quéré, Alan Rodger, and Stephen Griffies for useful advice and input that helped improve the original draft of this paper. We gratefully acknowledge the GFDL CM2.4 developing team for their modelling efforts. We also thank Jan Viebhan and Carsten Eden for providing us with the data from their numerical simulation. ACNG was supported by a NERC Advanced Research Fellowship (NE/C517633/1). AMH was supported by Australian Research Council Grant DP0877824. Numerical computations were conducted using the National Facility of the Australian National Computational Infrastructure and the NOAA/GFDL computing facilities.

\section{APPENDIX}

\section{Derivation of the Zonal Momentum Equation for an Outcropping Isopycnal Layer [(7)]}

The eastward momentum equation integrated around an arbitrary quasi-zonal circumpolar path (e.g., following a baroclinic streamfunction contour) for an adiabatic isopycnal layer may be written as (e.g., Ward and Hogg 2011)

$$
\langle(h u) v\rangle_{y}-\langle f h v\rangle+\left\langle h M_{x}\right\rangle=\frac{\langle\tau\rangle}{\rho_{0}} .
$$

Here $u$ and $v$ are the eastward and northward components of velocity, respectively; $h$ is the isopycnal layer thickness; and $M$ is the Montgomery potential. A time average is assumed, and near-surface diabatic effects have been neglected as they are not expected to significantly affect the overall momentum balance of the layer.

The four terms in (A1) may be identified as, respectively, 1) the northward flux of eastward momentum due to Reynolds stresses; 2) the Coriolis force; 3 ) pressure gradient effects, which govern the net form drag; and 4) the contribution of surface wind stress to eastward momentum over the outcrop area of the isopycnal layer. The Reynolds stress term has been repeatedly found to be a small contributor to the ACC momentum balance (e.g., Stevens and Ivchenko 1997) and may thus be ignored to first order. Equation (A1) is thus simplified to

$$
-\langle f h v\rangle+\left\langle h M_{x}\right\rangle=\frac{\langle\tau\rangle}{\rho_{0}} .
$$

Now a Reynolds decomposition of the pressure gradient term into mean and eddy (primed) components allows us to eliminate the mean component (since $\left\langle M_{x}\right\rangle=0$ ), leaving just the eddy component. Assuming that eddies are close to geostrophic, $M_{x}^{\prime}=f v^{\prime}$, and thus (A2) may be simplified to

$$
-\langle f v h\rangle+\left\langle f v^{\prime} h^{\prime}\right\rangle-\frac{\langle\tau\rangle}{\rho_{0}} \approx 0,
$$

where an approximate equivalence between meridional and cross-stream (i.e., across the quasi-zonal circumpolar path of integration or averaging) velocities has been invoked. Note that the latter approximation may not hold in regions of large stationary meanders, but this should not affect the differential analysis in expressions (9)-(11) if the ACC path remains fixed. Thus, we opt for retaining this generalization. Next, we define the Ertel potential vorticity (Ertel 1942) of the isopycnal layer as $q=(f+\zeta) / h$, where $\zeta$ is the vertical component of the relative vorticity. Assuming that $\zeta \ll f$ and that $h^{\prime} \ll\langle h\rangle$, as appropriate to quasigeostrophic dynamics, the potential vorticity perturbation with respect to a spatial and temporal mean may then be expressed as

$$
q^{\prime}=\frac{f h^{\prime}}{\langle h\rangle^{2}}+\frac{f^{\prime}}{\langle h\rangle}
$$

If the first term on the right-hand side of (A4) is assumed to be dominant, as would occur in the absence of large meridional excursions of the ACC [again, this assumption is not critical to the differential analysis in expressions (9)-(11) if the ACC path remains fixed], (A3) may be rewritten as

$$
-\langle f v h\rangle+\langle h\rangle^{2}\left\langle v^{\prime} q^{\prime}\right\rangle-\frac{\langle\tau\rangle}{\rho_{0}} \approx 0 .
$$


Dividing by $\langle f\rangle\langle h\rangle$, we obtain our reference expression for the zonal momentum balance of an outcropping isopycnal layer [i.e., (7) in the main text]:

$$
-\frac{\langle f v h\rangle}{\langle f\rangle\langle h\rangle}=\frac{\langle h\rangle\left\langle v^{\prime} q^{\prime}\right\rangle}{\langle f\rangle}-\frac{\langle\tau\rangle}{\left(\langle f\rangle \rho_{0}\langle h\rangle\right)} .
$$

\section{REFERENCES}

Allison, L. C., H. L. Johnson, and D. P. Marshall, 2011: Spin-up and adjustment of the Antarctic Circumpolar Current and global pycnocline. J. Mar. Res., in press.

Böning, C. W., A. Dispert, M. Visbeck, S. R. Rintoul, and F. U. Schwarzkopf, 2008: The response of the Antarctic Circumpolar Current to recent climate change. Nat. Geosci., 1 (12), 864-869.

Danabasoglu, G., and J. Marshall, 2007: Effects of vertical variations of thickness diffusivity in an ocean general circulation model. Ocean Modell., 18, 122-141.

Eden, C., and R. Greatbatch, 2008: Towards a mesoscale eddy closure. Ocean Modell., 20, 223-239.

Ertel, H., 1942: Ein neuer hydrodynamischer Wirbelsatz (A new hydrodynamic vorticity equation). Meteor. Z., 59 (9), $277-$ 281.

Farneti, R., and T. L. Delworth, 2010: The role of mesoscale eddies in the remote oceanic response to altered Southern Hemisphere winds. J. Phys. Oceanogr., 40, 2348-2354.

—, and P. Gent, 2011: The effects of the eddy-induced advection coefficient in a coarse-resolution coupled climate model. Ocean Modell., 39 (1-2), 135-145, doi:10.1016/j.ocemod.2011. 02.005 .

— , T. L. Delworth, A. J. Rosati, S. M. Griffies, and F. Zeng, 2010: The role of mesoscale eddies in the rectification of the Southern Ocean response to climate change. J. Phys. Oceanogr., 40, 1539-1557.

Ferrari, R., and M. Nikurashin, 2010: Suppression of eddy mixing across jets in the Southern Ocean. J. Phys. Oceanogr., 40, $1501-1519$

Fyfe, J. C., and O. A. Saenko, 2006: Simulated changes in the extratropical Southern Hemisphere winds and currents. Geophys. Res. Lett., 33, L06701, doi:10.1029/2005GL025332.

Gent, P. R., and J. C. McWilliams, 1990: Isopycnal mixing in ocean circulation models. J. Phys. Oceanogr., 20, 150-155.

—_, and G. Danabasoglu, 2011: Response to increasing Southern Hemisphere winds in CCSM4. J. Climate, 24, 49924998.

Griffies, S. M., and Coauthors, 2005: Formulation of an ocean model for global climate simulations. Ocean Sci., 2, 165-246.

Hallberg, R., and A. Gnanadesikan, 2006: The role of eddies in determining the structure and response of the wind-driven Southern Hemisphere overturning: Results from the Modeling Eddies in the Southern Ocean (MESO) project. J. Phys. Oceanogr., 36, 2232-2252.

Hofmann, M., and M. A. Morales Maqueda, 2011: The response of Southern Ocean eddies to increased midlatitude westerlies: A non-eddy resolving model study. Geophys. Res. Lett., 38, L03605, doi:10.1029/2010GL045972.

Hogg, A. M., 2010: An Antarctic Circumpolar Current driven by surface buoyancy forcing. Geophys. Res. Lett., 37, L23601, doi:10.1029/2010GL044777.
— W. Dewar, P. Killworth, and J. Blundell, 2003: A quasigeostrophic coupled model: Q-GCM. Mon. Wea. Rev., 131, 2261-2278.

Ito, T., M. Woloszyn, and M. Mazloff, 2010: Anthropogenic carbon dioxide transport in the Southern Ocean driven by Ekman flow. Nature, 463 (7277), 80-83.

Key, R. M., and Coauthors, 2004: A global ocean carbon climatology: Results from Global Data Analysis Project (GLODAP). Global Biogeochem. Cycles, 18, GB4031, doi:10. 1029/2004GB002247.

Law, R., R. J. Matear, and R. J. Francey, 2008: Comment on "Saturation of the Southern Ocean $\mathrm{CO}_{2}$ sink due to recent climate change." Science, 319 (5863), doi:10.1126/science.1149077.

Le Quéré, C., and Coauthors, 2007: Saturation of the Southern Ocean $\mathrm{CO}_{2}$ sink due to recent climate change. Science, 316 (5831), 1735-1738.

— of the Southern Ocean $\mathrm{CO}_{2}$ sink due to recent climate change." Science, 319, doi:10.1126/science.1147315.

Marshall, D. P., and A. Adcroft, 2010: Parameterization of ocean eddies: Vorticity mixing, energetics and Arnold's first stability theorem. Ocean Modell., 32, 188-204.

Marshall, G. J., 2003: Trends of the Southern Ocean $\mathrm{CO}_{2}$ sink due to recent climate change. J. Climate, 16, 1734-1738.

Marshall, J., and T. Radko, 2003: Residual-mean solutions for the Antarctic Circumpolar Current and its associated overturning circulation. J. Phys. Oceanogr., 33, 2341-2354.

Meredith, M. P., and A. M. Hogg, 2006: Circumpolar response of Southern Ocean eddy activity to a change in the Southern Annular Mode. Geophys. Res. Lett., 33, L16608, doi:10.1029/ 2006GL026499.

, P. L. Woodworth, C. W. Hughes, and V. Stepanov, 2004: Changes in the ocean transport through Drake Passage during the 1980s and 1990s, forced by changes in the Southern Annular Mode. Geophys. Res. Lett., 31, L21305, doi:10.1029/ 2004GL02169.

Morrison, A. K., A. M. Hogg, and M. L. Ward, 2011: Sensitivity of Southern Ocean overturning circulation to surface buoyancy forcing. Geophys. Res. Lett., 38, L14602, doi:10.1029/ 2011 GL048031.

Morrow, R., M. L. Ward, S. Pasquet, and A. M. Hogg, 2010: Eddy response to Southern Ocean climate modes. J. Geophys. Res., 115, C10030, doi:10.1029/2009JC005894.

Naveira Garabato, A. C., R. Ferrari, and K. Polzin, 2011: Eddy stirring in the Southern Ocean. J. Geophys. Res., 116, C09019, doi:10.1029/2010JC006818.

Olbers, D., and V. O. Ivchenko, 2001: On the meridional circulation and balance of momentum in the Southern Ocean of POP. Ocean Dyn., 52, 79-93.

, and M. Visbeck, 2005: A model of the zonally averaged stratification and overturning in the Southern Ocean. J. Phys. Oceanogr., 35, 1190-1205.

Pollard, R. T., M. I. Lucas, and J. F. Read, 2002: Physical controls on biogeochemical zonation in the Southern Ocean. Deep-Sea Res. II, 49 (16), 3289-3305.

Salmon, R., 1998: Lectures on Geophysical Fluid Dynamics. Oxford University Press, $378 \mathrm{pp}$.

Sen Gupta, A., and M. H. England, 2006: Coupled oceanatmosphere-ice response to variations in the Southern Annular Mode. J. Climate, 19, 4457-4486.

Smith, W. H. F., and D. T. Sandwell, 1997: Global seafloor topography from satellite altimetry and ship depth soundings. Science, 277, 195-196. 
Solomon, S., D. Qin, M. Manning, M. Marquis, K. Averyt, M. M. B. Tignor, H. L. Miller Jr., and Z. Chen, Eds., 2007: Climate Change 2007: The Physical Science Basis. Cambridge University Press, $996 \mathrm{pp}$.

Speer, K., S. R. Rintoul, and B. Sloyan, 2000: The diabatic Deacon cell. J. Phys. Oceanogr., 30, 3212-3222.

Stevens, D. P., and V. O. Ivchenko, 1997: The zonal momentum balance in an eddy-resolving general circulation model of the southern ocean. Quart. J. Roy. Meteor. Soc., 123, 929-951.

Straub, D. N., 1993: On the transport and angular momentum balance of channel models of the Antarctic Circumpolar Current. J. Phys. Oceanogr., 23, 776-782.

Taylor, G. I., 1921: Diffusion by continuous movements. Proc. London Math. Soc., 20, 196-212.

Thompson, D. W. L., and S. Solomon, 2002: Interpretation of recent Southern Hemisphere climate change. Science, 296, 895-899.
Treguier, A. M., J. L. Sommer, J. M. Molines, and B. de Cuevas, 2010: Response of the Southern Ocean to the Southern Annular Mode: Interannual variability and multidecadal trend. J. Phys. Oceanogr., 40, 1659-1668.

Vallis, G. K., 2006: Atmospheric and Oceanic Fluid Dynamics: Fundamentals and Large-Scale Circulation. Cambridge University Press, $745 \mathrm{pp}$.

Viebahn, J., and C. Eden, 2010: Towards the impact of eddies on the response of the Southern Ocean to climate change. Ocean Modell., 34, 150-165.

Ward, M. L., and A. M. Hogg, 2011: Establishment of momentum balance by form stress in a wind-driven channel. Ocean Modell., 40, 133-146, doi:10.1016/j.ocemod.2011.08.004.

Zickfeld, K., J. C. Fyfe, M. Eby, and A. J. Weaver, 2008: Comment on "Saturation of the Southern Ocean $\mathrm{CO}_{2}$ sink due to recent climate change." Science, 319 (570b), doi:10.1126/science. 1146886. 